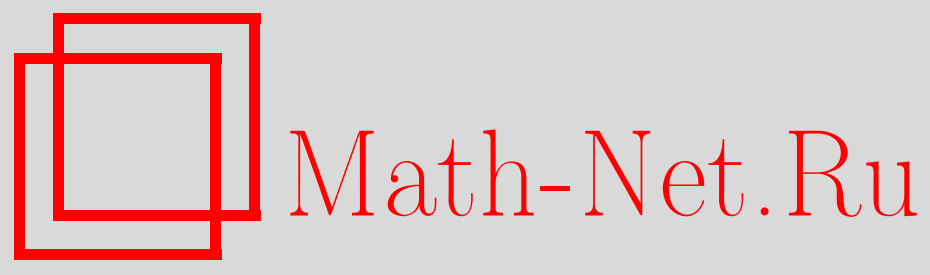

И. Б. Симоненко, Предельные теоремы типа Сегё для операторов обобщенной дискретной свертки, Матем. заметкu, 2005, том 78, выпуск 2, 265-277

DOI: https://doi.org/10.4213/mzm2584

Использование Общероссийского математического портала Math-Net.Ru подразумевает, что вы прочитали и согласны с пользовательским соглашением http://www.mathnet.ru/rus/agreement

Параметры загрузки:

IP: 54.166 .219 .16

26 апреля 2023 г., 12:33:25

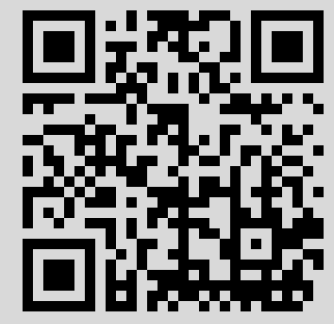




\section{ПРЕДЕЛЬНЫЕ ТЕОРЕМЫ ТИПА СЕГЁ ДЛЯ ОПЕРАТОРОВ ОБОБЩЕННОЙ ДИСКРЕТНОЙ СВЕРТКИ}

\section{И. Б. Симоненко}

Изучено асимптотическое поведение усредненного $f$-следа усеченного оператора обобщенной многомерной дискретной свертки при расшшрении области усечения. По определению усредненный $f$-след конечномерного оператора $A$ равен $n^{-1} \times$ $\sum_{k=1}^{n} f\left(\lambda_{k}\right)$, где $n$ - размерность пространства, в котором действует оператор $A$, $\lambda_{k}, k=1, \ldots, n,-$ полный набор собственных чисел оператора $A$ с учетом их кратности; обобщенная дискретная свертка - это оператор из замыкания алгебры, порожденной операторами дискретной свертки и операторами умножения на функции, допускающие непрерьвное продолжение на бесконечно удаленную cферер.

Библиографоия: 15 названий.

Интерес автора к данной проблеме возник под влиянием известной работы Сегё [1], где рассмотрены одномерные дискретные свертки (по этому поводу см. также [2, гл. 5] и [3, с. 167-172]). Наиболее близкими к настоящей работе являются статьи [4], [5]. В [4] изучено асимптотическое поведение определителей усеченных многомерных дискретных сверток с положительными символами. В работе [5], недавно выполненной автором, так же, как и в настоящей статье, изучены операторы обобщенной многомерной дискретной свертки, т.е. операторы из алгебры, порожденной многомерными дискретными свертками и операторами умножения на функции, допускающие непрерьвное продолжение на бесконечно удаленную сферу. Однако в работе [5] на функцию $f$ (см. теоремы 1.2 и 1.4 настоящей работы) наложены более сильные ограничения, что связано со следуюшим обстоятельством. В [5] сначала рассматривается случай, когда $f$ - полином, а затем функции $f$, которые можно аппроксимировать полиномами в области, где конщентрируется спектр усечений. В настоящей работе сначала рассматривается случай, ког да $f(z)=\left(z-z_{0}\right)^{-1}$, а затем функции $f$, которыеможно аппроксимировать в той же области, но рациональными функциями. В связи с этим в данной работе большую роль играет возможность почти явно построить оператор, обратньй к усеченному, и провести его локальный анализ. Это удается сделать, пользуясь методами, развитыми в работах [6]-[8].

1. Основные результаты. Пусть $\mathbb{Z}, \mathbb{C}, \mathbb{N}$ - множества целых, комплексных и натуральных чисел соответственно; $n \in \mathbb{N} ;$ множество $\Xi \stackrel{\text { def }}{=} \mathbb{Z}^{n}$ снабжено дискретной мерой, принимающей значение 1 на каждом одноэлементном множестве; $l_{2}=L_{2}(\Xi)$; $S=\{z|z \in \mathbb{C}| z \mid,=1\} ; \Theta=S^{n}$ - тор, снабженньй инвариантной относительно естественной групповой операции мерой $\operatorname{mes} \Theta$, удовлетворяющей условию $\operatorname{mes}_{\Theta}(\Theta)=1$; 
$L_{2}=L_{2}(\Theta) ; F: l_{2} \rightarrow L_{2}$ - оператор Лорана-Фурье, т.е. оператор, определяемьй равенством

$$
(F g)(\theta)=\sum_{\xi \in \Xi} g(\xi) \theta^{\xi}
$$

где $\theta^{\xi}=\left(\theta_{1}, \ldots, \theta_{n}\right)^{\left(\xi_{1}, \ldots, \xi_{n}\right)}=\theta_{1}^{\xi_{1}} \cdots \theta_{n}^{\xi_{n}}$.

Обозначим через $\mathfrak{C}$ подалгебру алгебры $\operatorname{End}\left(l_{2}\right)$, состоящую из таких операторов $C$, что $F C F^{-1}$ - оператор умножения на некоторую функцию $m$ из $\mathbb{C}(\Theta)$; такую функцию $m$ обозначим через $\mu(C)$. Здесь и далее, $\operatorname{End}(B)$ и $\mathbb{C}(X)$, где $B$ - банахово, а $X-$ компактное топологическое пространство, - банаховы алгебры соответственно линейных ограниченных операторов, действующих в пространстве $B$, и комплекснозначных непрерьвных функций, определенных на $X$.

Пусть $\mathbb{R}$ - множество вещественных чисел; $\Omega=\mathbb{R}^{n}-n$-мерное нормированное арифметическое пространство; $\Omega_{1}=\{x \mid x \in \Omega,\|x\|=1\} ; \mathfrak{M}_{1}$ - алгебра определенных на $\Xi$ функций $m$, допускающих продолжение на бесконечно удаленную сферу, т.е. таких, что для каждой точки $x_{0} \in \Omega_{1}$ существует конечный предел $\lim m(x)$, когда $x /\|x\| \rightarrow x_{0}$ и $\|x\| \rightarrow+\infty ; \mathfrak{M} \subset \operatorname{End}\left(l_{2}\right)$ - алгебра операторов умножения на функции, принадлежащие $\mathfrak{M}_{1}$.

Определим, наконец, алгебру $\mathfrak{G}$. Эта алгебра является замыканием в $\operatorname{End}\left(l_{2}\right)$ алгебры, порожденной алгебрами $\mathfrak{C}$ и $\mathfrak{M}$. Известно (см. [9], [10]), что существует и притом только один непрерывный гомоморфизм $\sigma: \mathfrak{G} \rightarrow \mathbb{C}\left(\Theta \times \Omega_{1}\right)$, удовлетворяющий следующему условию: для каждых $C \in \mathfrak{C}, m \in \mathfrak{M}_{1}, \theta \in \Theta, \omega \in \Omega_{1}$ имеет место равенство

$$
[\sigma(C M)](\theta, \omega)=[(\mu(C))(\theta)] m_{\infty}(\omega)
$$

где $M$ - оператор умножения на функцию $m ; m_{\infty}-$ функция, определенная на $\Omega_{1}$ следующим образом:

$$
m_{\infty}(\omega)=\lim _{\xi /\|\xi\| \rightarrow \omega,\|\xi\| \rightarrow+\infty} m(\xi) .
$$

Пусть всюду далее $A \in \mathfrak{G}, \nu=\sigma(A) ; D$ - ограниченное измеримое по Жордану (см. в [11] статью “Жордана мера") подмножество пространства $\Omega ; V$ - мера Лебега в $\mathbb{R}^{n} ; V(D)>0 ; x_{0} \in \Omega ; \vartheta_{\alpha}: \Omega \rightarrow \Omega, \alpha>0,-$ отображение, определяемое равенством $\vartheta_{\alpha}(x)=x_{0}+\alpha\left(x-x_{0}\right) ; \Xi_{\alpha}=\Xi \cap \vartheta_{\alpha}(D), \alpha>0 ; L_{2}(X), X \subset \Xi,-$ подпространство пространства $l_{2}$, состоящее из тех функций, которые обращаются в нуль вне $X$; $j_{X}: L_{2}(X) \subset l_{2}, P_{X}: l_{2} \rightarrow L_{2}(X)$ - операторы вложения и умножения на характеристическую функцию множества $X$ соответственно; $l_{2}^{\alpha}=L_{2}\left(\Xi_{\alpha}\right) ; A_{\alpha} \in \operatorname{End}\left(l_{2}^{\alpha}\right)$ - оператор, заданный соотношением $A_{\alpha}=P_{\Xi_{\alpha}} A j_{\Xi_{\alpha}} ; K(u)=\bigcup_{\alpha \geqslant 0} \vartheta_{\alpha}\left(u+x_{0}\right), u \subset \Omega_{1} ; L=\bigcap_{l \in \mathfrak{A}} l$, где $\mathfrak{A}$ - множество тех замкнутых подмножеств $l$ множества $\Omega_{1}$, для которых $K(l) \supset D$; $G_{\omega}, \omega \in \Omega_{1},-$ вьпуклая оболочка множества $\{z \mid z=\nu(\theta, \omega), \theta \in \Theta\} ; \Gamma=\bigcup_{\omega \in L} G_{\omega}$.

Теорема 1.1. Пусть $x_{0}$ не принадлежит замыканию множества $D ; G$ - открытое подмнохсество пространства $\mathbb{C}$, содерхсащее в себе Г. Тогда существует такое $\alpha_{0}>0$, что для каждого $\alpha>\alpha_{0}$ все собственные числа оператора $A_{\alpha}$ находятся в $G$.

Нам понадобятся еще следующие обозначения. Пусть $\operatorname{Def}(f)$ - область определения отображения $f ; \mathbb{R}^{+}$- множество положительных вещественных чисел; $\operatorname{Numb}(X)$ - количество элементов во множестве $X ; n_{\alpha}=\operatorname{Numb}\left(\Xi_{\alpha}\right), \alpha \in \mathbb{R}^{+} ; \Phi_{f, G}: \mathbb{R}^{+} \rightarrow \mathbb{C}$, когда 
$f$ - комплекснозначная функция и $G \subset \operatorname{Def}(f) \subset \mathbb{C}$ - функция, определяемая формулой

$$
\Phi_{f, G}(\alpha)=n_{\alpha}^{-1} \sum_{j \in N} f\left(\zeta_{j}\right),
$$

где $\zeta_{1}, \ldots, \zeta_{n_{\alpha}}$-полный набор собственных чисел оператора $A_{\alpha}$ с учетом их кратности; $N$ - множество тех $j \in\left\{1, \ldots, n_{\alpha}\right\}$, для которых $\zeta_{j} \in G ; \Phi_{f}=\Phi_{f, \operatorname{Def}(f)} ; V_{1}$ - мера, определенная на измеримых по Лебегу подмножествах сферы $\Omega_{1}$ равенством

$$
V_{1}(u)=V[K(u) \cap D] / V(D) .
$$

ТЕОРема 1.2. Пусть выполняются условия теоремы 1.1, $f$ - аналитическая функция комплексного переменного, определенная в $G$. Тогда

$$
\Phi_{f}(\alpha) \underset{\alpha \rightarrow+\infty}{\longrightarrow} I_{f} \stackrel{\text { def }}{=} \int_{\Theta \times L} f(\nu) d\left(\text { mes } \Theta \times V_{1}\right) .
$$

И, наконец, последние обозначения которые понадобятся нам в данном пункте: $\Gamma_{0}=$ $\mathbb{C} \backslash \bigcup_{H \in \mathfrak{H}} H$, где $\mathfrak{H}$ - множество тех компонент связности множества $\mathbb{C} \backslash \bigcup_{\omega \in \Omega_{1}} G_{\omega}$, которые содержат по крайней мере одну точку, не принадлежащую спектру оператора $A$.

Tеорема 1.3. Пусть $x_{0}$ - внутренняя точка множества $D ; G$ - открытое подмножество $\mathbb{C}$, содержащие в себе $\Gamma_{0}$. Тогда существуют такие $\alpha_{0}>0$ и $M>0$, что для каждого $\alpha>\alpha_{0}$ число точек спектра оператора $A_{\alpha}$, расположсенных вне $G$, с учетом их кратности не превосходит $M$.

Теорема 1.4. Пусть выполняются условия теоремы 1.3, $f$ - аналитическая функиия комплексного переменного, определенная в $G, G_{1}$ - открытое подмножество комплексной плоскости, содержащее в себе $\Gamma_{0}$, и замыкание $G_{1}$ вложено в G. Тогда

$$
\Phi_{f, G_{1}}(\alpha) \underset{\alpha \rightarrow+\infty}{\longrightarrow} I_{f}^{0}=\int_{\Theta \times \Omega_{1}} f(\nu) d\left(\operatorname{mes}_{\Theta} \times V_{1}\right) .
$$

Доказательство сформулированных теорем содержится в следуюших трех пунктах.

2. Доказательство теоремы 1.1. Пусть $\bar{A}_{\omega}, \omega \in \Omega_{1},-$ оператор из $\mathfrak{C}$, удовлетворяющий условию: для всех $\theta \in \Theta$ вьполнено равенство $\left[\mu\left(\bar{A}_{\omega}\right)\right](\theta)=\nu(\theta, \omega) ; E_{X}-$ единичный оператор в алгебре $\operatorname{End}\left(L_{2}(X)\right) ; j_{X, Y}: L_{2}(X) \subset L_{2}(Y), P_{Y, X}: L_{2}(Y) \rightarrow L_{2}(X)$, $X \subset Y \subset \Xi$,- операторы вложения и умножения на характеристическую функцию множества $X$ соответственно; $\iota_{B}$, когда $B$ - оператор из $\operatorname{End}\left[L_{2}(X)\right], X \subset \Xi,-$ функция, определенная на $\mathbb{R}^{+}$соотношением

$$
\iota_{B}(d)=\sup _{\left(X_{1}, X_{2}\right) \in \mathfrak{A}}\left\|P_{X, X_{1}} B j_{X_{2}, X}\right\|_{\operatorname{Hom}\left(L_{2}\left(X_{2}\right), L_{2}\left(X_{1}\right)\right)},
$$

где $\mathfrak{A}$ - множество таких пар $\left(X_{1}, X_{2}\right)$, что $X_{1} \subset \Xi, X_{2} \subset \Xi$ и

$$
\inf _{x_{1} \in X_{1}, x_{2} \in X_{2}}\left\|x_{1}-x_{2}\right\| \geqslant d .
$$

Здесь и далее, $\operatorname{Hom}\left(B_{1}, B_{2}\right)$, где $B_{1}, B_{2}-B$-пространства, - банахово пространство линейных ограниченных операторов, действующих из $B_{1}$ в $B_{2}$. 
ЛЕмма 2.1. Пусть $L_{1}$ - замкнутое подмножсество пространства $\Omega_{1} ; \lambda \in \mathbb{C} \backslash$ $\bigcup_{\omega \in L_{1}} G_{\omega} ; B_{X, \omega}=P_{X} \bar{A}_{\omega} j_{X}-\lambda E_{X}, X \subset \Xi, \omega \in L_{1}$, - семейство операторов. Тогда

1) все операторы этого семейства обратимы;

2) существует такое $C>0$, что для каждых $X \subset \Xi, \omega \in L_{1}$ имеет место оченка $\left\|B_{X, \omega}^{-1}\right\|_{\text {End } L_{2}(X)} \leqslant C$;

3) функиия $\varphi$, определенная на $\mathbb{R}^{+}$равенством

$$
\varphi(d)=\sup _{X \subset \Xi, \omega \in L_{1}}\left[\iota_{B_{X, \omega}^{-1}}\right](d),
$$

стремится к нулю при $d$, стремящемся $\kappa+\infty$.

Это утверж дение доказано в $[6$, с. 92$]$ и $[8$, с. 121 , следствие].

Введем обозначение $O(u, r), u \subset \Omega_{1}, r>0$ :

$$
O(u, r)=\left\{x \mid x \in \Omega, \frac{1}{\left\|x-x_{0}\right\|}\left(x-x_{0}\right) \in u,\left\|x-x_{0}\right\|>r\right\} .
$$

Лемма 2.2. Пусть $\xi \in \Omega_{1}$. Тогда для кажддого $\varepsilon>0$ существует такая окрестность и точки $\xi$ в пространстве $\Omega_{1}$ и такое $r>0$, что

$$
\left\|\left(A-\bar{A}_{\xi}\right) j_{U \cap \Xi}\right\|_{\mathrm{Hom}\left(l_{2}(U \cap \Xi), l_{2}\right)}<\varepsilon
$$

əде $U=O(u, r)$.

Это предложение содержится в [9].

Напомним определение кратности пересечения. Пусть $\mathfrak{X}=\left\{X_{\alpha} \mid \alpha \in \mathfrak{A}\right\}$ - семейство множеств. Обозначим через $r(x)$ число тех $\alpha \in \mathfrak{A}$, для которых $x \in X_{\alpha}$; через $X$ объединение $\bigcup_{\alpha \in \mathfrak{A}} X_{\alpha}$. Кратностью пересечения семейства $\mathfrak{X}$ назьвается $\sup _{x \in X} r(x)$.

Лемма 2.3. Пусть $X=\left(X_{1}, \ldots, X_{N}\right), Y=\left(Y_{1}, \ldots, Y_{N}\right)$ - семейства подмножеств множества $\Xi ; r, 1$ - кратности пересечения семейств $X, Y$ соответственно $\left(A_{1}, \ldots, A_{N}\right)$ - семейство операторов из $\operatorname{End}\left(l_{2}\right) ; c=\max _{i=1}^{N}\left\|A_{i}\right\|_{\operatorname{End}\left(l_{2}\right)}$. Тогда имеет место оченка

$$
\left\|\sum_{i=1}^{N} j_{Y_{i}} P_{Y_{i}} A_{i} j_{X_{i}} P_{X_{i}}\right\|_{\operatorname{End}\left(l_{2}\right)} \leqslant r c .
$$

Эта лемма содержится в [12, с. 27, лемма 2.1].

Последующая лемма используется при доказательстве как теоремы 1.1, так и теоремы 1.2. Ее формулировку и доказательство можно было бы существенно облегчить, если бы нам предстояло доказать лишь первую из них.

Для формулировки этой леммы нам понадобятся некоторые обозначения. Пусть $\mathbb{J}_{1}-$ кольцо измеримых по Жордану подмножеств сферы $\Omega_{1}$, т.е. таких подмножеств $u$ сферы $\Omega_{1}$, для которых пересечение $K(u)$ с любым ограниченным измеримым по Жордану подмножеством пространства $\Omega$ также измеримо по Жордану; $\operatorname{close}_{X}(Y)$, где $X$ - топологическое пространство, а $Y \subset X$, - замыкание $Y$ в $X$; $\phi$ - модуль непрерьвности функции $\nu$ (предполагается, что пространство $\Theta \times \Omega_{1} \subset \mathbb{C}^{n} \times \mathbb{R}^{n}$ снабжено естественной метрикой); $\operatorname{Diam}(X)$, где $X$ - метрическое пространство, - диаметр множества $X$; $I_{\alpha}=E_{\Xi_{\alpha}}$. 
Лемма 2.4. Пусть $\lambda \in \mathbb{C} \backslash \Gamma$. Тогда для каждого $\varepsilon>0$ существуют $N \in \mathbb{N}$, $r>0$, набор $\left(u_{i}, f_{i}, \xi_{i}\right), i=1, \ldots, N, u \alpha_{0}>0$ mакие, что

1) $\left\{u_{i}\right\},\left\{f_{i}\right\}, i=1, \ldots, N,-$ семейства подмножеств пространства $\Omega_{1}$, принадлежащих $\mathbb{J}_{1} ;$ причем каждое из әтих семейств образует покрытие $L$; их кратности пересечения не превосходят п и 1 соответственно;

2) для каждого $i \in\{1, \ldots, N\}$ множество $u_{i}$ - откритое подмножество пространства $\Omega_{1}$ и имеет место вложение сlose $\Omega_{1}\left(f_{i}\right) \subset u_{i}$;

3) $\xi_{i} \in L u$

$$
\phi(\delta)<\varepsilon, \quad \text { əдe } \delta=\sup _{i \in\{1, \ldots, N\}} \operatorname{Diam}\left(\left\{\xi_{i}\right\} \cup u_{i}\right)
$$

4)

$$
N\left(V_{1}\left(u_{i}\right)-V_{1}\left(f_{i}\right)\right)<\varepsilon
$$

5) для каждого $\alpha>\alpha_{0}$ имеет место оченка

$$
\left\|J-I_{\alpha}\right\|_{\operatorname{End}\left(l_{2}^{\alpha}\right)}<\varepsilon
$$

əдe

$$
\begin{gathered}
J=B\left(A_{\alpha}-\lambda I_{\alpha}\right) \\
B=\sum_{i=1}^{N} j_{F_{i} \cap \Xi_{\alpha}, \Xi_{\alpha}} P_{U_{i} \cap \Xi_{\alpha}, F_{i} \cap \Xi_{\alpha}}\left[P_{U_{i} \cap \Xi_{\alpha}}\left(\bar{A}_{\xi_{i}}-\lambda E_{\Xi}\right) j_{U_{i} \cap \Xi_{\alpha}}\right]^{-1} P_{\Xi_{\alpha}, U_{i} \cap \Xi_{\alpha}} \\
U_{i}=O\left(u_{i}, r\right), \quad F_{i}=O\left(f_{i}, r\right) .
\end{gathered}
$$

ДокаЗАТЕЛЬСтво. Пусть $\varepsilon>0$. Построение объектов, существование которых утверждается в лемме, проведем в несколько приемов.

I. Число $c_{1}$. Положим

$$
c_{1}=\sup _{X \subset \Xi, \xi \in L}\left\|\left(P_{X} \bar{A}_{\xi} j_{X}-\lambda E_{X}\right)^{-1}\right\|_{\operatorname{End}\left(L_{2}(X)\right)} .
$$

На основании леммы 2.1 можно гарантировать, что $c_{1}<+\infty$.

II. Числа $\delta, N, r ;$ наборы $\left(u_{1}, \ldots, u_{N}\right),\left(f_{1}, \ldots, f_{N}\right),\left(\xi_{1}, \ldots, \xi_{N}\right),\left(U_{1}, \ldots, U_{N}\right),\left(F_{1}\right.$, $\left.\ldots, F_{N}\right)$. Пусть $\delta$ - положительное число, удовлетворяющее условию $\phi(\delta)<\varepsilon$. В силу леммы 2.2 для каждого $\xi \in L$ существуют такие открытое подмножество $v_{\xi}$ сферы $\Omega_{1}$ и $r_{\xi}>0$, что
a) $\xi \in v_{\xi}$;
b) $\operatorname{Diam}\left(v_{\xi}\right)<\delta$;
c) $\left\|\left(A-\bar{A}_{\xi}\right) j_{O\left(v_{\xi}, r_{\xi}\right) \cap \Xi}\right\|_{\operatorname{Hom}\left(L_{2}\left(O\left(v_{\xi}, r_{\xi}\right) \cap \Xi\right), l_{2}\right)}<\left(2 n c_{1}\right)^{-1} \varepsilon$. 
Пусть $v \stackrel{\text { def }}{=}\left\{v_{\xi}\right\},\left\{r_{\xi}\right\}, \xi \in L,-$ такие семейства.

Пусть $N \in \mathbb{N}$ и наборы $u \stackrel{\text { def }}{=}\left\{u_{i}\right\},\left\{f_{i}\right\}, i=1, \ldots, N$, удовлетворяют условиям 1), 2), 4) и следующему дополнительному требованию: $u$ вписано в $v$, т.е. для каждого $i \in\{1, \ldots, N\}$ существует такое $\xi \in L$, что $u_{i} \subset v_{\xi}$. Пусть $\left(\xi_{1}, \ldots, \xi_{N}\right)$ - набор, удовлетворяющий условию $u_{i} \subset v_{\xi_{i}} ; r=\max _{i \in\{1, \ldots, N\}} r_{\xi_{i}} ; U_{i}=O\left(u_{i}, r\right), F_{i}=O\left(f_{i}, r\right)$. При этом, разумеется, выполняется и условие 3$)$.

III. Число $\alpha_{1}$. Пусть $\alpha_{1}>0$ - такое число, что для каждого $\alpha>\alpha_{1}$

$$
D_{\alpha} \stackrel{\text { def }}{=} \vartheta_{\alpha}(D) \subset \bigcup_{i=1}^{N} F_{i} .
$$

IV. Числа $d_{0}, \alpha_{2}$. Введем функцию $\iota^{\prime}$, определенную на $\mathbb{R}^{+}$равенством

$$
\iota^{\prime}(y)=\sup _{\alpha>0, i \in\{1, \ldots, N\}} \iota_{B_{\alpha, i}}(y),
$$

где

$$
B_{\alpha, i}=j_{U_{i} \cap \Xi_{\alpha}}\left[P_{U_{i} \cap \Xi_{\alpha}}\left(\bar{A}_{\xi_{i}}-\lambda E_{\Xi}\right) j_{U_{i} \cap \Xi_{\alpha}}\right]^{-1} P_{U_{i} \cap \Xi_{\alpha}}\left(A-\lambda E_{\Xi}\right) .
$$

Функция $\iota^{\prime}$ убывает и на основании леммы 2.1 настоящей работы и леммы 1 из [8] $\iota^{\prime}(y) \rightarrow 0$, когда $y \rightarrow+\infty$. Пусть $d_{0} \in \mathbb{R}^{+}, \alpha_{2}>0$ - такие числа, что $\iota^{\prime}\left(d_{0}\right)<(2 N)^{-1} \varepsilon$ и для каждых $\alpha>\alpha_{2}$ и $i \in\{1, \ldots, N\}$ имеет место оценка

$$
\inf _{x \in\left(\Omega \backslash U_{i}\right) \cap D_{\alpha}, y \in F_{i} \cap D_{\alpha}}\|x-y\|>d_{0} .
$$

$\mathrm{V}$. Оценка (2.3). Положим $\alpha_{0}=\max \left(\alpha_{1}, \alpha_{2}\right)$ и покажем, что при $\alpha>\alpha_{0}$ имеет место оценка (2.3).

Итак, пусть $\alpha>\alpha_{0}$. Пусть $J, B$ - операторы, определяемые формулами $(2.4),(2.5)$,

$$
\begin{aligned}
J_{1}= & \sum_{i=1}^{N} j_{F_{i} \cap \Xi_{\alpha}, \Xi_{\alpha}} P_{U_{i} \cap \Xi_{\alpha}, F_{i} \cap \Xi_{\alpha}}\left[P_{U_{i} \cap \Xi_{\alpha}}\left(\bar{A}_{\xi_{i}}-\lambda E_{\Xi}\right) j_{U_{i} \cap \Xi_{\alpha}}\right]^{-1} \\
& \times P_{\Xi \alpha, U_{i} \cap \Xi_{\alpha}} P_{\Xi_{\alpha}}\left(A-\lambda E_{\Xi}\right) j_{U_{i} \cap \Xi_{\alpha}} P_{U_{i} \cap \Xi_{\alpha}} j_{\Xi_{\alpha}} \\
J_{2}= & \sum_{i=1}^{N} j_{F_{i} \cap \Xi_{\alpha}, \Xi_{\alpha}} P_{U_{i} \cap \Xi_{\alpha}, F_{i} \cap \Xi_{\alpha}}\left[P_{U_{i} \cap \Xi_{\alpha}}\left(\bar{A}_{\xi_{i}}-\lambda E_{\Xi}\right) j_{U_{i} \cap \Xi_{\alpha}}\right]^{-1} \\
& \times P_{\Xi_{\alpha}, U_{i} \cap \Xi_{\alpha}} P_{\Xi_{\alpha}}\left(\bar{A}_{\xi_{i}}-\lambda E_{\Xi}\right) j_{U_{i} \cap \Xi_{\alpha}} P_{U_{i} \cap \Xi_{\alpha}} j_{\Xi_{\alpha}} .
\end{aligned}
$$

Положим

$$
a_{1}=\left\|J-J_{1}\right\|_{\operatorname{End}\left(l_{2}^{\alpha}\right)}, \quad a_{2}=\left\|J_{1}-J_{2}\right\|_{\operatorname{End}\left(l_{2}^{\alpha}\right)}, \quad a_{3}=\left\|J_{2}-I_{\alpha}\right\|_{\operatorname{End}\left(l_{2}^{\alpha}\right)} .
$$

Покажем, что $a_{1}, a_{2}<\varepsilon / 2, a_{3}=0$.

Сначала оцениваем $a_{3}$.

Принимая во внимание, что

$$
\begin{aligned}
J_{2}= & \sum_{i=1}^{N} j_{F_{i} \cap \Xi_{\alpha}}, \Xi_{\alpha} P_{U_{i} \cap \Xi_{\alpha}, F_{i} \cap \Xi_{\alpha}}\left[P_{U_{i} \cap \Xi_{\alpha}}\left(\bar{A}_{\xi_{i}}-\lambda E_{\Xi}\right) j_{U_{i} \cap \Xi_{\alpha}}\right]^{-1} \\
& \times P_{U_{i} \cap \Xi_{\alpha}}\left(\bar{A}_{\xi_{i}}-\lambda E_{\Xi}\right) j_{U_{i} \cap \Xi_{\alpha}} P_{U_{i} \cap \Xi_{\alpha}} j_{\Xi_{\alpha}}
\end{aligned}
$$




$$
\begin{aligned}
& =\sum_{i=1}^{N} j_{F_{i} \cap \Xi_{\alpha}, \Xi_{\alpha}} P_{U_{i} \cap \Xi_{\alpha}, F_{i} \cap \Xi_{\alpha}} P_{U_{i} \cap \Xi_{\alpha}} j_{\Xi_{\alpha}}=\sum_{i=1}^{N} j_{F_{i} \cap \Xi_{\alpha}, \Xi_{\alpha}} P_{F_{i} \cap \Xi_{\alpha}} j_{\Xi_{\alpha}} \\
& =\sum_{i=1}^{N} j_{F_{i} \cap \Xi_{\alpha}, \Xi_{\alpha}} P_{\Xi_{\alpha}, F_{i} \cap \Xi_{\alpha}} P_{\Xi_{\alpha}} j_{\Xi_{\alpha}}
\end{aligned}
$$

и $\alpha>\alpha_{1}$, получаем

$$
\sum_{i=1}^{N} j_{F_{i} \cap \Xi_{\alpha}, \Xi_{\alpha}} P \Xi_{\alpha}, F_{i} \cap \Xi_{\alpha}=I_{\alpha},
$$

откуда и вытекает равенство $a_{3}=0$.

Оценка $a_{2}<\varepsilon / 2$ вытекает из леммы 2.3 .

Оценим $a_{1}$. В соответствии с определением $J, J_{1}$ имеем

$$
\begin{aligned}
J-J_{1}= & \sum_{i=1}^{N} j_{F_{i} \cap \Xi_{\alpha}, \Xi_{\alpha}} P_{U_{i} \cap \Xi_{\alpha}, F_{i} \cap \Xi_{\alpha}}\left[P_{U_{i} \cap \Xi_{\alpha}}\left(\bar{A}_{\xi_{i}}-\lambda E_{\Xi}\right) j_{U_{i} \cap \Xi_{\alpha}}\right]^{-1} \\
& \times P_{U_{i} \cap \Xi_{\alpha}}\left(A-\lambda E_{\Xi}\right) j_{\left(\Omega \backslash U_{i}\right) \cap \Xi_{\alpha}} P_{\left(\Omega \backslash U_{i}\right) \cap \Xi_{\alpha}} j_{\Xi_{\alpha}} .
\end{aligned}
$$

Поскольку $\alpha>\alpha_{2}$, получаем оценку $a_{1}<\varepsilon / 2$.

Лемма 2.5. Пусть $\lambda \in \mathbb{C} \backslash \Gamma$. Тогда существуют такие $\alpha_{0}>0$ и $>>0$, что для каждого $\alpha>\alpha_{0}$ оператор $A_{\alpha}-\lambda I_{\alpha}$ обратим и имеет место оценка

$$
\left\|\left(A_{\alpha}-\lambda I_{\alpha}\right)^{-1}\right\|_{\operatorname{End}\left(l_{2}^{\alpha}\right)} \leqslant c .
$$

ДоказАтЕльство. Пусть $\varepsilon=1 / 2, N \in \mathbb{N}, r>0$, набор $\left(u_{i}, f_{i}, \xi_{i}\right), i=1, \ldots, N$, и $\alpha_{0}>0$ удовлетворяют условиям 1)-5) леммы 2.4. Пусть $c_{1}$ определяется формулой (2.6). Тогда, как это вытекает из лемм 2.4 и 2.3 , для каждого $\alpha>\alpha_{0}$ оператор $A_{\alpha}-\lambda I_{\alpha}$ обратим и справедливо неравенство

$$
\left\|\left(A_{\alpha}-\lambda I_{\alpha}\right)^{-1}\right\|_{\operatorname{End}\left(l_{2}^{\alpha}\right)} \leqslant 2 n c_{1} .
$$

Теорема 1.1 является следствием этой леммы и компактности множества Г.

\section{3. Доказательство теоремы 1.2 .}

Лемма 3.1. Пусть $\lambda \in \mathbb{C} \backslash \Gamma$. Тогда для кажсдого $\varepsilon>0$ существуют $N \in \mathbb{N}$, $r>0$, набор $\left(u_{i}, f_{i}, \xi_{i}\right), i=1, \ldots, N, u \alpha_{0}>0$ такие, что выполняются условия 1)-4) леммы 2.4 и вместо условия 5) выполняется следуюшее: для кажсдого $\alpha>\alpha_{0}$ оператор $A_{\alpha}-\lambda I_{\alpha}$ обратим и имеет место оценка

$$
\left\|B-\left(A_{\alpha}-\lambda I_{\alpha}\right)^{-1}\right\|_{\operatorname{End}\left(l_{2}^{\alpha}\right)}<\varepsilon,
$$

где $B$ - оператор, определяемый равенством (2.5).

Эта лемма является следствием лемм 2.4, 2.5. 
Лемма 3.2. Пусть $X \subset \Omega$ ограничено и измеримо по Жордану; $V(X)>0$; $X_{\alpha}=\Xi \cap \vartheta_{\alpha}(X), \alpha \in \mathbb{R}^{+} ; B \in \mathfrak{C} ; 0$ не принадлежит выпуклой оболочке образа функиии $\mu(B) ; B_{\alpha}=P_{X_{\alpha}} B j_{X_{\alpha}}, \alpha>0 ; \Psi-$ функиия, определенная на $\mathbb{R}^{+}$соотношением

$$
\Psi(\alpha)=m_{\alpha}^{-1} \sum_{j=1}^{m_{\alpha}} \frac{1}{\zeta_{j}},
$$

где $m_{\alpha}=\operatorname{Numb}\left(X_{\alpha}\right),\left\{\zeta_{1}, \ldots, \zeta_{m_{\alpha}}\right\}$ - полный набор собственных чисел оператора $B_{\alpha}$ с учетом их кратности (заметим, что нуля среди них нет). Тогда

$$
\Psi(\alpha) \underset{\alpha \rightarrow+\infty}{\longrightarrow} \int_{\Theta}\left[\frac{1}{\mu(B)}\right] d\left(\operatorname{mes}_{\Theta}\right) .
$$

Данное предложение доказано в [13] (случай, когда символ принадлежит классу Винера) и [5] (случай, когда символ непрерывен; теорема 3.1$)$.

Лемма 3.3. Пусть $X \subset \Omega-$ измеримо по Жордану и ограничено; $X_{\alpha}=\vartheta_{\alpha}(X)$ $\cap \Xi, \alpha>0 ; m_{\alpha}=\operatorname{Numb}\left(X_{\alpha}\right)$. Tогдa

$$
\frac{m_{\alpha}}{\alpha^{n}} \underset{\alpha \rightarrow+\infty}{\longrightarrow} V(X)
$$

Это предложение является непосредственньм следствием определения измеримого по Жордану множества.

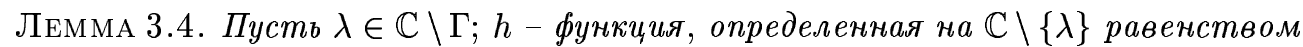
$h(z)=(z-\lambda)^{-1}$. Тогдa

$$
\Phi_{h}(\alpha) \underset{\alpha \rightarrow+\infty}{\longrightarrow} J \stackrel{\text { def }}{=} \int_{\Theta \times L} h(\nu) d\left(\operatorname{mes}_{\Theta} \times V_{1}\right) .
$$

ДокАЗАТЕЛЬСТво. Пусть $c_{1}$ определяется формулой $(2.6)$;

$$
c_{2}=\sup _{\theta \in \Theta, \xi \in L}|[h(\nu)](\theta, \xi)| ;
$$

$\omega_{h}$ - модуль непрерывности функции $h$ на $\Gamma$, т.е. $\omega_{h}-$ функция, определенная на $\mathbb{R}^{+}$равенством

$$
\omega_{h}(x)=\sup _{z_{1} \in \Gamma, z_{2} \in \Gamma,\left\|z_{1}-z_{2}\right\|<x}\left|h\left(z_{1}\right)-h\left(z_{2}\right)\right| .
$$

Пусть $\varepsilon>0$. Покажем, что существует такое $\alpha^{\prime}>0$, что для каждого $\alpha>\alpha^{\prime}$

$$
\left|\Phi_{h}(\alpha)-J\right|<2 \varepsilon+2 c_{1} \varepsilon+c_{2} \varepsilon+\omega_{h}(\varepsilon) .
$$

Выбор $\alpha^{\prime}$ проведем в несколько этапов.

I. Выбор $N, r,\left(u_{i}, f_{i}, \xi_{i}\right), i=1, \ldots, N, \alpha_{0}$. Пусть $N \in \mathbb{N}, r>0$, набор $\left(u_{i}, f_{i}, \xi_{i}\right)$, $i=1, \ldots, N$, и $\alpha_{0}>0$ выбраны так, что вьполняются условия леммы 3.1. Отметим, что при $\alpha>\alpha_{0}$ оператор $A_{\alpha}-\lambda I_{\alpha}$ обратим. 
II. Выбор $U_{i}, F_{i}, \nu_{\xi}$. Пусть

$$
U_{i}=O\left(u_{i}, r\right), \quad F_{i}=O\left(f_{i}, r\right) ;
$$

$\nu_{\xi}, \xi \in \Omega_{1},-$ функция, определенная на $\Theta$ равенством

$$
\nu_{\xi}(\theta)=[\nu(A)](\theta, \xi) .
$$

III. Выбор $J_{1}, \ldots, J_{5} ; a_{1}, \ldots, a_{5}$. Пусть $J_{3}, J_{4}, J_{5}, a_{4}, a_{5} \in \mathbb{C} ; J_{1}, J_{2}, a_{1}, a_{2}, a_{3}$ - функции, определенные на луче $\left(\alpha_{0},+\infty\right)$ следующим образом:

$$
\begin{gathered}
J_{1}(\alpha)=\frac{1}{n_{\alpha}} \operatorname{trac}\left[\sum_{i=1}^{N} j_{F_{i} \cap \Xi_{\alpha}, \Xi_{\alpha}} P_{U_{i} \cap \Xi_{\alpha}, F_{i} \cap \Xi_{\alpha}}\right. \\
\left.\times\left[P_{U_{i} \cap \Xi_{\alpha}}\left(\bar{A}_{\xi_{i}}-\lambda E_{\Xi}\right) j_{U_{i} \cap \Xi_{\alpha}}\right]^{-1} P_{\Xi_{\alpha}, U_{i} \cap \Xi_{\alpha}}\right], \\
J_{2}(\alpha)=\frac{1}{n_{\alpha}} \operatorname{trac}\left[\sum_{i=1}^{N} j_{U_{i} \cap \Xi_{\alpha}, \Xi_{\alpha}}\left[P_{U_{i} \cap \Xi_{\alpha}}\left(\bar{A}_{\xi_{i}}-\lambda E_{\Xi}\right) j_{U_{i} \cap \Xi_{\alpha}}\right]^{-1} P_{\Xi_{\alpha}, U_{i} \cap \Xi_{\alpha}}\right], \\
J_{3}=\sum_{i=1}^{N} V_{1}\left(u_{i}\right) \int_{\Theta} h\left(\nu_{\xi_{i}}\right) d \operatorname{mes}_{\Theta}, \quad J_{4}=\sum_{i=1}^{N} V_{1}\left(f_{i}\right) \int_{\Theta} h\left(\nu_{\xi_{i}}\right) d \operatorname{mes}_{\Theta}, \quad J_{5}=J, \\
a_{1}=\left|\Phi_{h}-J_{1}\right|, \quad a_{i}=\left|J_{i-1}-J_{i}\right|, \quad i=2, \ldots, 5 .
\end{gathered}
$$

Здесь и всюду далее, $\operatorname{trac}(C)$, где $C$ - конечномерный оператор, - след оператора $C$. IV. Оценка $a_{1}$. Учитывая, что

$$
\Phi_{h}(\alpha)=\frac{1}{n_{\alpha}} \operatorname{trac}\left(A_{\alpha}-\lambda I_{\alpha}\right)^{-1}, \quad J_{1}(\alpha)=\frac{1}{n_{\alpha}} \operatorname{trac} B,
$$

где $B$ определяется формулой (2.5), и оценку (3.1), заключаем, что при $\alpha>\alpha_{0}$ имеет место оценка $a_{1}(\alpha)<\varepsilon$.

V. Выбор $\alpha_{1}$. В силу леммы 3.3 существует такое $\alpha_{1}>\alpha_{0}$, что для каждых $\alpha>\alpha_{1}$ и $i \in\{1, \ldots, N\}$ вьполнено

$$
\left|\frac{\operatorname{Numb}\left(\left(U_{i} \backslash F_{i}\right) \cap \Xi_{\alpha}\right)}{n_{\alpha}}-V_{1}\left(u_{i} \backslash f_{i}\right)\right|<\frac{\varepsilon}{N} .
$$

Пусть $\alpha_{1}$ - такое число. Заметим, что из оценки (2.2) следует, что при $\alpha>\alpha_{1}$ имеет место неравенство

$$
\sum_{i=1}^{N} \frac{\operatorname{Numb}\left(\left(U_{i} \backslash F_{i}\right) \cap \Xi_{\alpha}\right)}{n_{\alpha}} \leqslant 2 \varepsilon .
$$

VI. Оценка $a_{2}$. Учитывая, что

$$
\begin{aligned}
& J_{1}(\alpha)=\frac{1}{n_{\alpha}} \operatorname{trac}\left[\sum_{i=1}^{N} P_{U_{i} \cap \Xi_{\alpha}, F_{i} \cap \Xi_{\alpha}}\left[P_{U_{i} \cap \Xi_{\alpha}}\left(\bar{A}_{\xi_{i}}-\lambda E_{\Xi}\right) j_{U_{i} \cap \Xi_{\alpha}}\right]^{-1} j_{F_{i} \cap \Xi_{\alpha}, U_{i} \cap \Xi_{\alpha}}\right], \\
& J_{2}(\alpha)=\frac{1}{n_{\alpha}} \operatorname{trac}\left(\sum_{i=1}^{N}\left[P_{U_{i} \cap \Xi_{\alpha}}\left(\bar{A}_{\xi_{i}}-\lambda E_{\Xi}\right) j_{U_{i} \cap \Xi_{\alpha}}\right]^{-1}\right),
\end{aligned}
$$


имеем

$$
\begin{aligned}
a_{2}(\alpha)=\frac{1}{n_{\alpha}} \mid \operatorname{trac}\left(\sum_{i=1}^{N}\right. & P_{U_{i} \cap \Xi_{\alpha},\left(U_{i} \backslash F_{i}\right) \cap \Xi_{\alpha}} \\
& \left.\times\left[P_{U_{i} \cap \Xi_{\alpha}}\left(\bar{A}_{\xi_{i}}-\lambda E_{\Xi}\right) j_{U_{i} \cap \Xi_{\alpha}}\right]^{-1} j_{\left(U_{i} \backslash F_{i}\right) \cap \Xi_{\alpha}, U_{i} \cap \Xi_{\alpha}}\right) \mid .
\end{aligned}
$$

Отсюда и из (3.2) вытекает оценка $a_{2}(\alpha)<2 \varepsilon c_{1}$, справедливая при $\alpha>\alpha_{1}$.

VII. Выбор $\alpha_{2}$ и оценка $a_{3}$. Принимая во внимание леммы $3.2,3.3$, заключаем, что существует такое $\alpha_{2}>\alpha_{1}$, что для каждого $\alpha>\alpha_{2}$ выполнена оценка $a_{3}(\alpha)<\varepsilon$. Пусть $\alpha_{2}$ - такое число.

VIII. Оценка $a_{4}$. В силу (2.2) для каждого $\alpha>\alpha_{2}$ выполнена оценка $a_{4}(\alpha) \leqslant \varepsilon c_{2}$.

IX. Оценка $a_{5}$. В силу оценки (2.1) для каждого $i \in\{1, \ldots, N\}$ выполнено

$$
\left|V_{1}\left(f_{i}\right) \int_{\Theta}\left[h\left(\nu_{\xi_{i}}\right)\right] d \operatorname{mes}_{\Theta}-\int_{\Theta \times f_{i}}[h(\nu)] d\left(\operatorname{mes} \Theta \times V_{1}\right)\right| \leqslant \omega_{h}(\varepsilon) V_{1}\left(f_{i}\right) .
$$

Отсюда вытекает оценка $a_{5} \leqslant \omega_{h}(\varepsilon)$.

Х. Окончательная оценка. Таким образом, при $\alpha>\alpha_{2}$ вьполнено

$$
\left|\Phi_{h}-J\right| \leqslant a_{1}+a_{2}+a_{3}+a_{4}+a_{5}<\varepsilon+2 \varepsilon c_{1}+\varepsilon+\varepsilon c_{2}+\omega_{h}(\varepsilon) .
$$

Лемма 3.5. Пусть $K$ - замкнутое ограниченное подмножество плоскости $\mathbb{C}$; $H$ - открытое подмнохество $\mathbb{C} ; K \subset H ; h$ - определенная на $H$ аналитическая функиия комплексного переменного. Тогда для каждого $\varepsilon>0$ существуют такие $m \in \mathbb{N}, c_{k} \in \mathbb{C} u z_{k} \in \mathbb{C} \backslash K, k=1, \ldots, m$, ито

$$
\sup _{z \in K}\left|h(z)-\sum_{j=1}^{m} c_{k}\left(z-z_{k}\right)^{-1}\right|<\varepsilon
$$

Эта лемма является следствием, например, теоремы 8 из [14, с. 28].

Теперь нетрудно завершить доказательство теоремы 1.2.

Пусть $\varepsilon>0$. Покажем, что сушествует такое $\alpha_{0}$, что для каждого $\alpha>\alpha_{0}$ вьполнена оценка

$$
\left|\Phi_{f}(\alpha)-I_{f}\right|<\varepsilon
$$

Пусть $G_{1}$ - открытое ограниченное подмножество комплексной плоскости; $\bar{G}_{1}=$ $\operatorname{close}_{\mathbb{C}}\left(G_{1}\right) ; \Gamma \subset G_{1}, \bar{G}_{1} \subset G ; R-$ функция вида

$$
R(z)=\sum_{k=1}^{m} c_{k}\left(z-z_{k}\right)^{-1}
$$

где $c_{k} \in \mathbb{C}, z_{k} \in \mathbb{C} \backslash \bar{G}_{1}$, такая, что

$$
\sup _{z \in \bar{G}_{1}}|f(z)-R(z)|<\frac{\varepsilon}{3} .
$$


Такая функция существует в силу леммы 3.5 .

Пусть

$$
J=\int_{\Theta \times L} R(\nu) d\left(\operatorname{mes}_{\Theta} \times V_{1}\right) .
$$

В силу лемм $2.5,3.4$ существует такое $\alpha_{0}>0$, что для каждого $\alpha>\alpha_{0}$ :

а) для каждого $\lambda \in \mathbb{C} \backslash \bar{G}_{1}$ оператор $A_{\alpha}-\lambda I_{\alpha}$ обратим;

б) вьполняется оценка

$$
\left|\Phi_{R}(\alpha)-J\right|<\frac{\varepsilon}{3}
$$

Пусть $\alpha_{0}-$ такое число.

Тогда при $\alpha>\alpha_{0}$

$$
\left|\Phi_{f}(\alpha)-I_{f}\right| \leqslant\left|\Phi_{f}(\alpha)-\Phi_{R}(\alpha)\right|+\left|\Phi_{R}(\alpha)-J\right|+\left|J-I_{f}\right|<\frac{\varepsilon}{3}+\frac{\varepsilon}{3}+\frac{\varepsilon}{3}=\varepsilon .
$$

4. Доказательство теорем 1.3 и 1.4. Пусть $\operatorname{Spectr}(C)$, где $C \in \operatorname{End}(B), B$ - некоторое банахово пространство,- спектр оператора $C ; K_{z_{0}, r}=\left\{z|z \in \mathbb{C}| z-,z_{0} \mid<r\right\}$, $z_{0} \in \mathbb{C}, r>0$.

Лемма 4.1. Пусть $\lambda_{0} \in\left(\mathbb{C} \backslash \Gamma_{0}\right) \cap \operatorname{Spectr}(A)$. Тогда

1) $\lambda_{0}$ - изолированное собственное число оператора $A$ конечной кратности;

2) существует такое $r>0$, что для любого $\varepsilon \in(0, r)$ существует такое $\alpha_{0}>0$, что для каждого $\alpha>\alpha_{0}$

$$
\operatorname{Spectr}\left(A_{\alpha}\right) \cap K_{\lambda_{0}, r}=\operatorname{Spectr}\left(A_{\alpha}\right) \cap K_{\lambda_{0}, \varepsilon}
$$

и число собственных чисел оператора $A_{\alpha}$ с учетом их кратности в каждом из этих мнохеств равно кратности собственного числа $\lambda_{0}$ оператоpa $A$.

ДокАЗАТЕльство. Существует такое связное открытое подмножество $H$ комплексной плоскости, что для каждого $\lambda \in H$ оператор $A-\lambda E_{\Xi}$ является оператором Нётеpa, $\lambda_{0} \in H$ и в $H$ существует такое $\lambda$, что оператор $A-\lambda E_{\Xi}$ обратим. Поэтому, как хорошо известно (см., например, $[15$, с. 273] или [5, теорема 11.1], доказательство теоремы 11.1 проведено максимально подробно), $\lambda_{0}$ является изолированным собственным числом оператора $A$. Поскольку $A-\lambda_{0} E_{\Xi}$ - оператор Нётера, $\lambda_{0}$ является для оператора $A$ собственным числом конечной кратности [5, теорема 11.4]. Таким образом, пункт 1) доказан.

Пункт 2) вытекает отсюда и из теоремы 12.2 из [5].

Лемма 4.2. Пусть $\lambda_{0} \in\left(\mathbb{C} \backslash \Gamma_{0}\right) \backslash \operatorname{Spectr}(A)$. Тогда существуют такие $c>0 u$ $\alpha_{0}>0$, что для каждого $\alpha>\alpha_{0}$ оператор $A_{\alpha}-\lambda_{0} I_{\alpha}$ обратим и имеет место оценка

$$
\left\|\left(A_{\alpha}-\lambda_{0} I_{\alpha}\right)^{-1}\right\|_{\operatorname{End}\left(l_{2}^{\alpha}\right)} \leqslant c .
$$

ДоКАЗАТЕЛЬСТВо аналогично доказательству леммы 2.5.

Теорема 1.3 вытекает из лемм 4.1, 4.2.

Приступим к доказательству теоремы 1.4.

Не уменьшая обшности, будем считать $G_{1}$ ограниченным. 
Лемма 4.3. Пусть $\lambda_{0} \in\left(\mathbb{C} \backslash \Gamma_{0}\right) \backslash \operatorname{Spectr}(A) ; h-$ функиия, определенная на $\mathbb{C} \backslash\left\{\lambda_{0}\right\}$ равенством $h(z)=\left(z-\lambda_{0}\right)^{-1}$. Тогда

$$
\Phi_{h}(\alpha) \underset{\alpha \rightarrow+\infty}{\longrightarrow} \int_{\Theta \times \Omega_{1}} h(\nu) d\left(\operatorname{mes}_{\Theta} \times V_{1}\right) .
$$

Эта лемма доказьвается так же, как и лемма 3.4.

Лемма 4.4. Пусть $m$ - число собственных чисел оператора $A$, расположснных в $\mathbb{C} \backslash G_{1}$, с учетом их кратности. Тогда $m<+\infty$ и существует такое $\alpha_{1}>0$, что для каждого $\alpha>\alpha_{1}$ число собственных чисел оператора $A_{\alpha}$, расположенных в $\mathbb{C} \backslash G_{1}$, с учетом их кратности не превосходит $\mathrm{m}$.

Этот факт вытекает из лемм 4.1 и 4.2.

Пусть $m$ - число собственных чисел оператора $A$, расположенных в $\mathbb{C} \backslash G_{1}$, с учетом их кратности; $\alpha_{1}>0$ - число, сушествование которого утверждается в лемме 4.4 .

ЛЕмма 4.5. Существуют ограниченные замкнутые подмноэсества $K_{1}, K_{2}$ комплексной плоскости и число $\alpha_{2}>\alpha_{1}$, удовлетворяющие следующим условиям:

1) $K_{1} \cap K_{2}=\varnothing$;

2) $G_{1} \subset K_{1} \subset G$;

3) для кажсдых $\alpha>\alpha_{2}, \lambda \in \mathbb{C} \backslash\left(K_{1} \cup K_{2}\right)$ оператор $A_{\alpha}-\lambda I_{\alpha}$ обратим.

Эта лемма также вытекает из лемм 4.1, 4.2.

Пусть $K_{1}, K_{2}, \alpha_{2}$ - объекты, существование которых утверждается в лемме 4.5 .

Лемма 4.6. Для каждого $\varepsilon>0$ существуют такие $M \in \mathbb{N}, c_{k} \in \mathbb{C} u z_{k} \in \mathbb{C} \backslash$ $\left(K_{1} \cup K_{2}\right), k=1, \ldots, M$, чmo

$$
\sup _{z \in K_{1}}\left|f(z)-\sum_{k=1}^{M} c_{k}\left(z-z_{k}\right)^{-1}\right|<\varepsilon .
$$

ДокАЗАТЕЛЬСТво. Введем функцию $h$, определенную на $K_{1} \cup K_{2}$ равенствами $\left.h\right|_{K_{1}}$ $=\left.f\right|_{K_{1}},\left.h\right|_{K_{2}}=0$. Функция $h$ допускает аналитическое продолжение в некоторое открытое подмножество, содержашее $K_{1} \cup K_{2}$. Используя лемму 3.5 , убеждаемся в справедливости нашего положения.

Теперь мы готовы завершить доказательство теоремы 1.4.

Пусть $\varepsilon>0 ; M, c_{k}, z_{k}, k=1, \ldots, M$, - объекты, существование которых утверждается в лемме $4.6 ; R$ - рациональная функция, заданная формулой

$$
\begin{gathered}
R(z)=\sum_{k=1}^{M} c_{k}\left(z-z_{k}\right)^{-1} ; \\
c=\sup _{z \in K_{1} \cup K_{2}}|R(z)|(\text { заметим, что } c<+\infty) ;
\end{gathered}
$$

$$
J=\int_{\Theta \times \Omega_{1}} R(\nu) d\left(\operatorname{mes}_{\Theta} \times V_{1}\right)
$$


$\alpha_{3}>\alpha_{2}$ - такое число, что для каждого $\alpha>\alpha_{3}$ вьполнено $\left|\Phi_{R}(\alpha)-J\right|<\varepsilon$. Такое число сушествует в силу леммы 4.3 .

Далее заметим, что при $\alpha>\alpha_{3}$

$$
\left|\Phi_{f, G_{1}}(\alpha)-\Phi_{R, G_{1}}(\alpha)\right|<\varepsilon, \quad\left|\Phi_{R, G_{1}}(\alpha)-\Phi_{R}(\alpha)\right|<\frac{m c}{n_{\alpha}}, \quad\left|J-I_{f}^{0}\right|<\varepsilon .
$$

Таким образом, мы доказали, что для каждого $\varepsilon>0$ существует такое $\alpha_{3}$, что для каждого $\alpha>\alpha_{3}$ имеет место неравенство

$$
\left|\Phi_{f, G_{1}}(\alpha)-I_{f}^{0}\right|<3 \varepsilon+\frac{m c}{n_{\alpha}}
$$

откуда и вытекает теорема 1.4.

\section{СПИСОК ЦИТИРОВАННОЙ ЛИТЕРАТУРЫ}

[1] Szegö G. On certain Hermitian forms associated with the Fourier series of positive functions // Festschrift Marcel Riesz. Lund, 1952. P. 228-238.

[2] Гренандер У., Сегё Г. Теплицевы формы и их приложения. М.: ИЛ, 1961.

[3] Böttcher A., Silbermann B. Invertibility and Asymptotics of Toeplitz Matrices. Berlin: Akademie-Verlag, 1983.

[4] Линник И. Ю. Многомерный аналог предельной теоремы Г. Сегё // Изв. АН СССР. Сер. матем. 1975. Т. 39. №6. С. 1393-1403.

[5] Симоненко И. Б. Предельные теоремы типа Сегё для операторов составной и обобщенной свертки // Деп. ВИНИТИ 12.08.99 № 2639-В99. Ростов-на-Дону, 1999.

[6] Гохберг И. Ц., Фельдман И. А. Уравнения в свертках и проекционные методы их решения. М.: Наука, 1971.

[7] Козак А. В. Локальный принцип в теории проекционных методов // Докл. АН СССР. 1973. T. 212. №6. C. 1287-1289.

[8] Козак А. В., Симоненко И. Б. Проекционные методы решения многомерных дискретных уравнений в свертках // Сиб. матем. ж. 1980. Т. 21. № 2. С. 119-127.

[9] Симоненко И. Б. О многомерных дискретных свертках // Математические исследования. Т. 3. № 1. Кишинев: Штиинца, 1968. С. 108-122.

[10] Симоненко И. Б. Операторы типа свертки в конусах // Матем. сб. 1967. Т. 74(116). № 2. C. $298-313$.

[11] Математическая энциклопедия. М.: Советская энциклопедия, 1979.

[12] Симоненко И. Б., Чинь Нгок Минь. Локальный метод в теории одномерных сингулярных интегральных уравнений с кусочно-непрерывными коэфффициентами. Нётеровость. Ростов-на-Дону: Изд-во РГУ, 1986.

[13] Симоненко И. Б. Многомерный вариант теоремы Г. Сегё о предельной плотности спектра усеченных операторов Теплица. Случай, когда символ принадлежит классу Винера // Деп. ВИНИТИ 21.08.98. № 2635-В98. Ростов-на-Дону, 1998.

[14] Уолш Дж.Л. Интерполяция и аппроксимация рациональными функциями в комплексной области. М.: ИЛ, 1961.

[15] Садовничий В. А. Теория операторов. М.: Изд-во МГУ, 1986.

Ростовский государственный университет

Поступило

E-mail: isim@ns.math.rsu.ru

03.04 .2000

Исправленный вариант

26.05 .2004 